\title{
ON EXPONENTIABILITY OF ÉTALE ALGEBRAIC HOMOMORPHISMS
}

\author{
MARIA MANUEL CLEMENTINO, DIRK HOFMANN, AND GEORGE JANELIDZE
}

\begin{abstract}
In this paper we show that the theorem, by Cagliari and Mantovani, stating that in the category of compact Hausdorff spaces every étale map is exponentiable, can be formulated in a general category $\operatorname{Alg}(T)$ of Eilenberg-Moore $T$-algebras, for a monad $T$, and proved in case $T$ satisfies the so-called Beck-Chevalley condition. For that, $\operatorname{Alg}(T)$ is embedded in the (topological) category $\operatorname{RelAlg}(T)$ of relational $T$-algebras, where a suitable notion of étale morphism can be studied, it is shown that morphisms between $T$-algebras are exponentiable in $\operatorname{RelAlg}(T)$, and, moreover, these exponentials belong to $\mathbf{A} \lg (T)$ whenever the morphisms are étale.
\end{abstract}

\section{INTRODUCTION}

The existence of "internal function objects" in a category $\mathbf{C}$ with finite products, that is the existence of a right adjoint to the functor ()$\times X: \mathbf{C} \rightarrow \mathbf{C}$ for a $\mathbf{C}$-object $X$ - calling then $X$ exponentiable -, is a widely studied problem. It is in general more interesting in topology than in algebra, since in a pointed category only the zero object is exponentiable. However, there is an interesting complete characterization of exponentiable objects in varieties by Johnstone [16]. Using monads instead of algebraic theories to study varieties, here we consider exponentiable morphisms in $\mathbf{C}$, that is, morphisms $f: A \rightarrow B$ which are exponentiable as objects of the comma category $\mathbf{C} \downarrow B$. Our approach is based on results obtained in topological contexts (namely from $[22,2,3,12]$ ) and makes use of the embedding of the category of $T$-algebras in the topological category of relational $T$-algebras (as introduced by Barr [1], and studied further in $[7,13,11])$. This way, the characterization of exponentiable continuous maps in the category of compact Hausdorff spaces as the local homeomorphisms, or étale maps, obtained by Cagliari and Mantovani in [2], can be formulated in any category of (Eilenberg-Moore) $T$-algebras - replacing the ultrafilter monad by a general monad $T$-, raising the question whether exponentiability in categories of $T$-algebras is more interesting at the level of morphisms than at the object level. This is the starting point of the work presented here.

In this paper we begin to study the exponentiability problem for the ordinary $T$-algebras for an arbitrary monad $T$ on the category of sets, assuming, as in [11], that $T$ satisfies the Beck-Chevalley Condition. After recalling all necessary definitions and results, we show that:

- When $f$ is a perfect map of relational $T$-algebras, its pseudo-relational exponents are relational, making it exponentiable in the category of relational $T$-algebras (Theorem 4.2). The notion of perfect used there, as well as the notion of étale used later, and related notions of open and proper, is suggested by the topological one as expected (see Section 2).

2010 Mathematics Subject Classification. 18C15, 18C20, 18D15, $18 \mathrm{~B} 30$.

Key words and phrases. exponentiable morphism, Eilenberg-Moore algebra, relational algebra, ultrafilter monad.

Research partially supported by Centro de Matemática da Universidade de Coimbra, by Centro de Investigação e Desenvolvimento em Matemática e Aplicações da Universidade de Aveiro/FCT, by Fundação para a Ciência e Tecnologia, through European program COMPETE/FEDER, by the projects MONDRIAN (under the contract PTDC/EIA-CCO/108302/2008) and PTDC/MAT/120222/2010, and by South African NRF. 
- Every homomorphism of $T$-algebras is perfect, and therefore exponentiable in the category of relational $T$-algebras (Corollary 4.3).

- When a homomorphism of $T$-algebras is étale, its (pseudo-)relational exponents are $T$ algebras, making it exponentiable in the category of $T$-algebras (Theorem 5.5).

In the last section we consider several examples of categories of algebras, namely, of compact Hausdorff spaces, sup-lattices, continuous lattices, monoids, semigroups, and monoid actions. In particular, we point out that, for a monoid $M$, although every morphism of $M$-sets is (well-known to be) exponentiable, not every morphism is étale - unless $M$ is a group.

In summary, we show that the exponentiability of étale maps in CompHaus $=\mathbf{A} \lg (U)$, with exponentials built as in Top $=\operatorname{RelAlg}(U)$, for $U$ the ultrafilter monad, can be generalized for $\operatorname{Alg}(T)$ in case $T$ satisfies the Beck-Chevalley condition. It remains to be shown whether the converse is true. The example of $M$-Set shows that there may be non-étale exponentiable morphisms in $\mathbf{A l g}(T)$, but in this example the exponentials, that exist both in $\operatorname{Alg}(T)$ and in $\operatorname{RelAlg}(T)$, are built differently.

The general problems of describing:

(I) exponentiable morphisms of relational $T$-algebras;

(II) exponentiable morphisms of $T$-algebras;

(III) exponentiable morphisms of $T$-algebras with exponents inherited from the category of relational $T$-algebras

remain open, but it seems that Proposition 4.1 should be helpful in solving Problem (II) (see also [12]), while étale maps should provide, in many cases, an answer to Problem (III). Our results on Problem (II) that do not use relational algebras, which is a work in progress now, will be published elsewhere. In particular, we will give an easy proof of the fact that a group homomorphism is exponentiable if and only if it is an isomorphism, and that the same is true in any semi-abelian category.

Acknowledgment. We thank an anonymous referee for pointing out a missing argument in the proof of Theorem 2.1.

\section{Relational algebras}

Given a monad $T=(T, \eta, \mu)$ on Set, we consider the category $\mathbf{A} \lg (T)$ of Eilenberg-Moore $T$-algebras; recall that an object of $\operatorname{Alg}(T)$ is a pair $(X, \alpha)$, where $X$ is a set and $\alpha: T X \rightarrow X$ is a map making the diagram

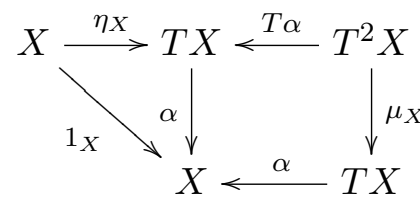

commute, and a morphism $f:(X, \alpha) \rightarrow(Y, \beta)$ is a map $f: X \rightarrow Y$ with $f \cdot \alpha=\beta \cdot T f$ :

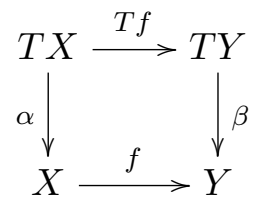


The monad $T$ can be extended to the 2-category Rel of relations (see $[1,8]$ ) as follows: for a relation $r: X \longrightarrow Y$, with $r=r_{2} \cdot r_{1}^{\circ}$,

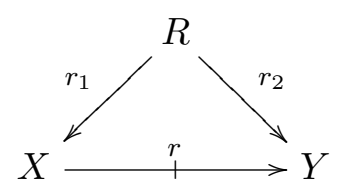

where $r_{1}, r_{2}$ are the projections and $r_{1}^{\circ}$ is the opposite relation of $r_{1}$, let $\overline{T r}=T r_{2} \cdot\left(T r_{1}\right)^{\circ}$. Then $\bar{T}: \mathbf{R e l} \rightarrow$ Rel is an op-lax functor, and $\eta: \operatorname{Id}_{\mathbf{R e l}} \rightarrow \bar{T}$ and $\mu: \bar{T}^{2} \rightarrow \bar{T}$ become op-lax natural transformations: for relations $r: X \mapsto Y$ and $s: Y \longrightarrow Z, \bar{T}(s \cdot r) \leq \bar{T}(s) \cdot \bar{T}(r)$, and $e_{Y} \cdot r \leq \bar{T}(r) \cdot e_{X}, m_{Y} \cdot \overline{T T}(r) \leq \bar{T}(r) \cdot m_{X}$. The functor $\bar{T}: \mathbf{R e l} \rightarrow \mathbf{R e l}$ is a lax - hence a strict - functor if and only if $T$ : Set $\rightarrow$ Set satisfies the Beck-Chevalley condition (BC), that is $T$ preserves weak pullbacks (that is, $(\mathrm{BC})$-squares: see $[7,8]$ ). This implies, in particular, that $T$ preserves pullbacks along monomorphisms, that is, $T$ is taut (see [21]).

Throughout we assume that $T:$ Set $\rightarrow$ Set has $(B C)$ and that the natural transformation $\mu: T T \rightarrow T$ satisfies $(B C)$, meaning that, for every map $f: X \rightarrow Y$, the naturality diagram

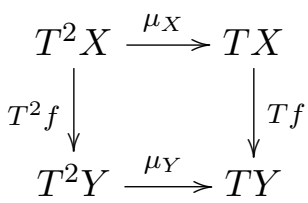

is a (BC)-square. We recall that these assumptions mean precisely that $\bar{T}: \mathbf{R e l} \rightarrow \mathbf{R e l}$ is a strict functor and that $\mu: \bar{T}^{2} \rightarrow \bar{T}$ is a strict natural transformation. We also assume that $T$ is non-trivial, or, equivalently, $T$ is faithful, or, equivalently, the natural transformation $\eta: \operatorname{Id} \rightarrow T$ is pointwise monic (see [17] for details).

As already studied by Barr [1] and studied later by Clementino, Hofmann and Tholen [7, 13, $11,8,9]$ and others, one can relax the conditions above, defining a relational $T$-algebra (also called lax T-algebra, or $(T, \mathbf{2})$-category) as a pair $(X, a)$, where $a: T X \mapsto X$ is a relation such that $1_{X} \leq a \cdot \eta_{X}$ and $a \cdot \bar{T} a \leq a \cdot \mu_{X}$, that is

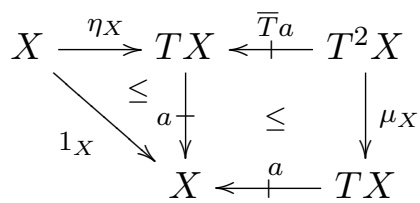

Morphisms $f:(X, a) \rightarrow(Y, b)$ between relational $T$-algebras are maps $f: X \rightarrow Y$ with $f \cdot a \leq$ $b \cdot T f:$

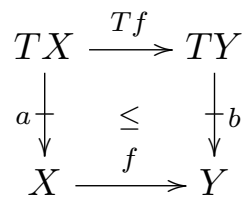

We will denote the category of relational $T$-algebras by $\operatorname{RelAlg}(T)$. Given a relational $T$-algebra $a: T X \mapsto X$, for $\mathfrak{x} \in T X$ and $x \in X$, we will write $\mathfrak{x} \rightarrow x$ whenever $\mathfrak{x} a x$. Using this notation, a relation $a: T X \mapsto X$ is a relational $T$-algebra if

(a) $(\forall x \in X) \eta_{X}(x) \rightarrow x$,

(b) $\left(\forall \mathfrak{X} \in T^{2} X\right)(\forall \mathfrak{x} \in T X)(\forall x \in X) \mathfrak{X} \rightarrow \mathfrak{x}$ and $\mathfrak{x} \rightarrow x \Rightarrow \mu_{X}(\mathfrak{X}) \rightarrow x$

(here $\mathfrak{X} \rightarrow \mathfrak{x}$ means $\mathfrak{X}(\bar{T} a) \mathfrak{x}$ ); a map $f: X \rightarrow Y$ is a morphism between the relational $T$-algebras $(X, a)$ and $(Y, b)$ if 
(c) $(\forall \mathfrak{x} \in T X)(\forall x \in X) \mathfrak{x} \rightarrow x \Rightarrow T f(\mathfrak{x}) \rightarrow f(x)$.

We remark that $\operatorname{Alg}(T)$ is fully embedded in $\operatorname{RelAlg}(T)$, since the inequality of diagram (A) becomes an equality whenever $a$ and $b$ are maps. Morphisms in $\operatorname{RelA} \lg (T)$ will be called simply homomorphisms, unless we want to identify those between $T$-algebras, calling them then algebraic homomorphisms.

We will make use also of a category containing $\operatorname{RelAlg}(T)$ as a full subcategory. A relation $a: T X \mapsto X$ is said to be a pseudo-relational $T$-algebra (also called lax reflexive $T$-algebra, or a $(T, \mathbf{2})-g r a p h)$ if $\eta_{X}(x) \rightarrow x$, that is:

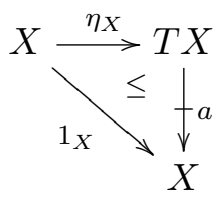

A morphism $f:(X, a) \rightarrow(Y, b)$ between pseudo-relational T-algebras is a map $f: X \rightarrow Y$ satisfying the inequality of diagram (A). Pseudo-relational $T$-algebras and their morphisms form the category PsRelAlg $(T)$. The full embeddings

$$
\operatorname{Alg}(T) \hookrightarrow \operatorname{RelAlg}(T) \hookrightarrow \text { PsRelAlg}(T) .
$$

have left adjoints; moreover, while $\mathbf{A l g}(T)$ is monadic over $\operatorname{Set}, \operatorname{RelAlg}(T)$ and $\operatorname{PsRelAlg}(T)$ are topological categories over Set (see [7] for details). In particular, the forgetful functor $\operatorname{Alg}(T) \rightarrow$ Set creates limits, while $\operatorname{RelAlg}(T) \rightarrow$ Set and PsRelAlg$(T) \rightarrow$ Set preserve limits and colimits.

\section{2. ÉtALE HOMOMORPHISMS}

In case $T$ is the ultrafilter monad, that is the monad induced by the adjunction

$$
\text { Set } \frac{\operatorname{Set}(, 2)}{\underset{\operatorname{Bool}(, 2)}{\longleftarrow}} \text { Bool }^{\text {op }}
$$

that assigns to each set $X$ its set $T X$ of ultrafilters, $T$-algebras are compact Hausdorff spaces (as shown by Manes [19]), relational $T$-algebras are topological spaces, homomorphisms between relational $T$-algebras are continuous maps (as shown by Barr [1]), and pseudo-relational algebras are pseudotopological spaces. This is the example that guides our approach to étale algebraic homomorphisms. It was shown in [6] that if a continuous map $f: X \rightarrow Y$ is étale, then, for each $x \in X$ and each ultrafilter $\mathfrak{y}$ with $\mathfrak{y} \rightarrow f(x)$, there exists a unique ultrafilter $\mathfrak{x} \in X$ such that $\mathfrak{x} \rightarrow x$ and $T f(\mathfrak{x})=\mathfrak{y}:$

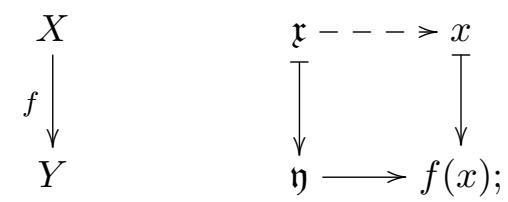

In [10] a continuous map with this property is called a discrete fibration, and it is shown that there are discrete fibrations which are not étale, and that étale maps are exactly the pullback-stable discrete fibrations.

Based on this example, a morphism $f:(X, a) \rightarrow(Y, b)$ between relational $T$-algebras will be said to be a discrete fibration if for every $x \in X$ and $\mathfrak{y} \in T Y$ with $\mathfrak{y} \rightarrow f(x)$ in $Y$, there exists a unique $\mathfrak{x} \in T X$ with $\mathfrak{x} \rightarrow x$ and $T f(\mathfrak{x})=\mathfrak{y}$. In particular, if $f:(X, \alpha) \rightarrow(Y, \beta)$ is an algebraic 
homomorphism, then $f$ is a discrete fibration if and only if the diagram

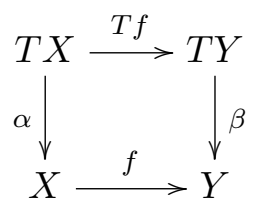

is a pullback. A pullback-stable discrete fibration in $\operatorname{RelAlg}(T)$ will be called étale. We remark that pullback stability of discrete fibrations along monomorphisms follows directly from (BC) of $T$.

Theorem 2.1. If $f:(X, \alpha) \rightarrow(Y, \beta)$ is an algebraic discrete fibration, then the following assertions are equivalent:

(i) $f$ is étale;

(ii) $f$ is a pullback-stable discrete fibration in $\mathbf{A} \lg (T)$;

(iii) for any algebraic homomorphism $g:(Z, \gamma) \rightarrow(Y, \beta)$, the pullback

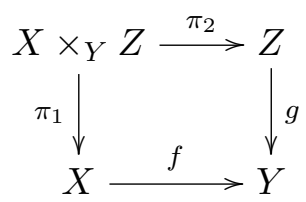

of $f$ along $g$ is preserved by $T$;

(iv) for any map $g: Z \rightarrow Y$, the pullback (B) of $f$ along $g$ is preserved by $T$.

Proof. (i) $\Rightarrow$ (ii) is trivial.

(ii) $\Leftrightarrow$ (iii): In the commutative diagram

(C)

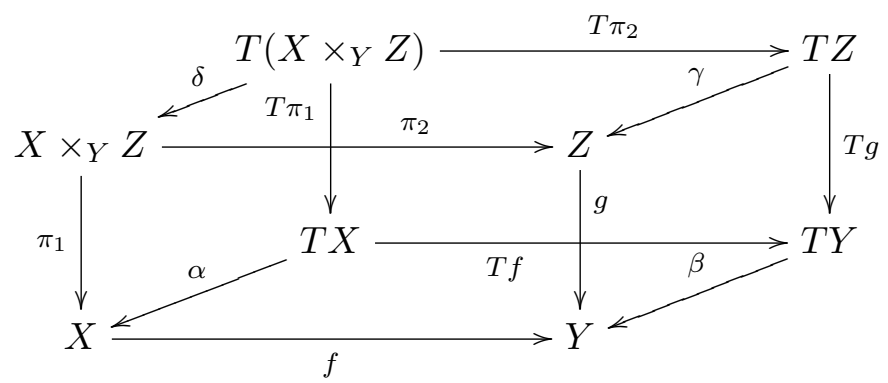

assume that the front face is a pullback. Closedness under limits of $\mathbf{A} \lg (T)$ guarantees that both $\pi_{1}$ and $\pi_{2}$ are algebraic homomorphisms. Since $f$ is a discrete fibration, the bottom square is also a pullback. Hence the top square is a pullback - that is, $\pi_{2}$ is a discrete fibration - if and only if the back square is a pullback, that is $T$ preserves the pullback of $f$ along $g$.

(iii) $\Leftrightarrow$ (iv): That (iv) $\Rightarrow$ (iii) is trivial. To show the converse, note that any map $g: Z \rightarrow Y$ factors through $\eta_{Z}: Z \rightarrow T Z$ via an algebraic homomorphism $\bar{g}:\left(T Z, \mu_{Z}\right) \rightarrow(Y, \beta)$. Hence preservation of pullback (B) by $T$ reduces to preservation of the pullback of $f$ along $\bar{g}$, which is guaranteed by (iii), and the preservation, by $T$, of the pullback

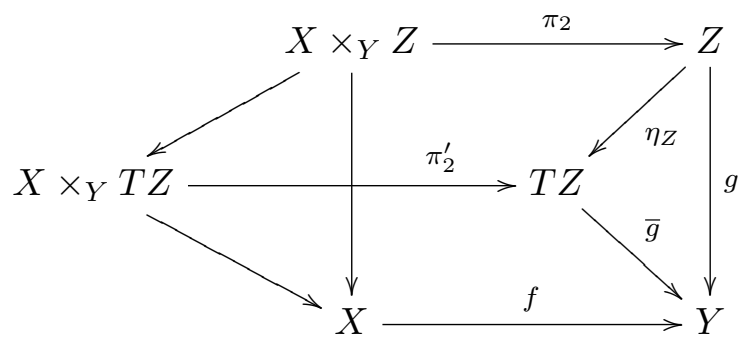


of $\pi_{2}^{\prime}$ along $\eta_{Z}$. Since $\eta_{Z}$ is monic, this follows from (BC) of $T$.

Finally to show (ii) $\Rightarrow$ (i) we consider any morphism $g:(Z, c) \rightarrow(Y, \beta)$ in $\operatorname{RelAlg}(T)$ and factor it as in diagram (D). By (ii) $\pi_{2}^{\prime}$ is a discrete fibration, and then $\pi_{2}$, as the pullback of a discrete fibration along a monomorphism, is a discrete fibration as well.

Corollary 2.2. (1) Every injective algebraic discrete fibration is étale.

(2) If the functor $T$ preserves pullbacks, then every algebraic discrete fibration is étale.

Following the characterization of proper, perfect and open continuous maps stated in $[6$, Theorem 2.2], in $[9,18]$ the following notions were studied in the context of relational $T$-algebras. A morphism $f:(X, a) \rightarrow(Y, b)$ is:

(a) open if for each $x \in X$ and $\mathfrak{y} \in T Y$ such that $\mathfrak{y} \rightarrow f(x)$ in $Y$, there exists $\mathfrak{x} \in T X$ such that $\mathfrak{x} \rightarrow x$ in $X$ and $T f(\mathfrak{x})=\mathfrak{y}$.

(b) proper (perfect) if for each $\mathfrak{x} \in T X$ and $y \in Y$ such that $T f(\mathfrak{x}) \rightarrow y$ in $Y$, there exists (a unique) $x \in X$ such that $\mathfrak{x} \rightarrow x$ and $f(x)=y$.

We remark that discrete fibrations are open, and that, for $f:(X, a) \rightarrow(Y, b)$ and diagrams

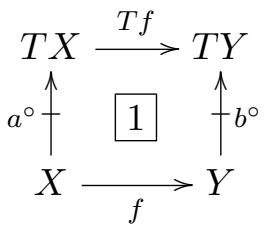

and

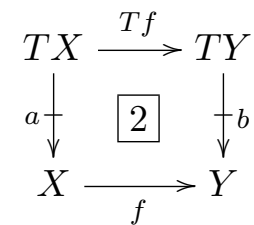

$f$ is open (resp. proper) if and only if diagram 1 (resp. 2 ) is commutative. In particular, every algebraic homomorphism is proper. It is in fact perfect because unicity of $x$ in (b) follows from the fact that $a$ is a map, so that $x=a(\mathfrak{x})$.

Finally we introduce some categories that will be used in the sequel. In our general setting we will say that a pseudo-relational $T$-algebra $(X, a)$ is Hausdorff if $a: T X \rightarrow X$ is a partial map, that is in its relation span

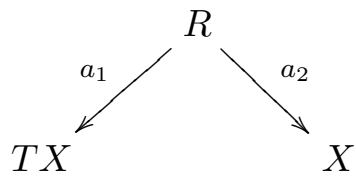

$a_{1}$ is injective. If $a_{1}$ is surjective, $(X, a)$ is said to be compact. It is easy to check that:

Proposition 2.3. If $(X, a)$ is a pseudo-relational T-algebra, then:

(1) $(X, a)$ is Hausdorff if and only if $\delta_{X}$ is proper;

(2) $(X, a)$ is compact if and only if $!_{X}: X \rightarrow 1$ is proper.

We denote by $\operatorname{Haus}(T)$ and $\operatorname{Comp}(T)$ the (full) subcategories of $\operatorname{RelA} \lg (T)$ of Hausdorff and compact relational $T$-algebras, respectively. We point out that $\operatorname{Alg}(T)$ is exactly the category of compact and Hausdorff relational $T$-algebras. It is a reflective subcategory of $\operatorname{RelAlg}(T)$, with the reflection constructed via the appropriate Stone-Čech compactification (see $[20,7]$ for details).

\section{Pseudo-Relational algebras form a quasitopos}

We recall that a category $\mathbf{C}$ is cartesian closed if it has finite products and every $\mathbf{C}$-object is exponentiable, and that $\mathbf{C}$ is said to be locally cartesian closed if, for every object $B$ of $\mathbf{C}$, the comma category $(\mathbf{C} \downarrow B)$ is cartesian closed, and $\mathbf{C}$ has a terminal object (and therefore 
all finite limits); $\mathbf{C}$ is a quasitopos if it is locally cartesian closed and it has a strong subobject classifier and all finite colimits.

When $\mathbf{C}$ is locally cartesian closed, and $f: A \rightarrow B$ is a morphism in $\mathbf{C}$, the right adjoint of the functor ()$\times(A, f):(\mathbf{C} \downarrow B) \rightarrow(\mathbf{C} \downarrow B)$ will be written as ()$^{(A, f)}$. In particular, for every object $C$ in $\mathbf{C}$, we have

$$
\left(B \times C, \mathrm{pr}_{1}\right)^{(A, f)}=\text { the partial product of } f \text { and } C .
$$

The original definition of (categorical) partial product, due to R. Dyckhoff and W. Tholen [15], is formulated as follows: the partial product of a morphism $f: A \rightarrow B$ and an object $C$ in a finitely complete category $\mathbf{C}$ is a pair $\left(p: P \rightarrow B, e: P \times_{B} A \rightarrow C\right)$ such that, given any pair of the form $\left(p^{\prime}: P^{\prime} \rightarrow B, e^{\prime}: P^{\prime} \times_{B} A \rightarrow C\right)$, there exists a unique $h: P^{\prime} \rightarrow P$ over $B$ with $e^{\prime}=e \cdot\left(h \times_{B} 1_{A}\right)$ in the diagram

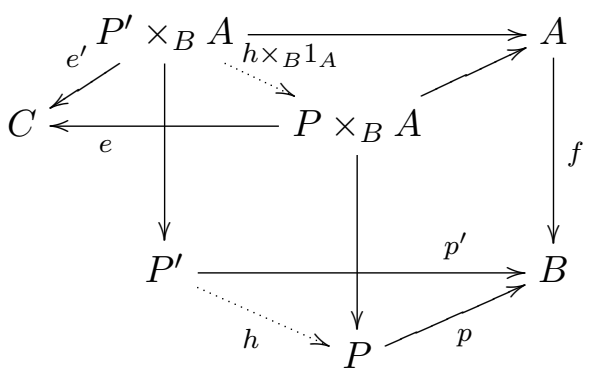

In fact $(P, p)=\left(B \times C, \mathrm{pr}_{1}\right)^{(A, f)}$, and $e$ determines the universal arrow

$$
() \times(A, f) \rightarrow\left(C \times B, \mathrm{pr}_{2}\right)
$$

as the pair $\left((P, p),\left\langle e, p \cdot \pi_{1}\right\rangle:(P, p) \times(A, f) \rightarrow\left(C \times B, \operatorname{pr}_{2}\right)\right)$, where $\pi_{1}: P \times_{B} A \rightarrow C$ is the pullback projection and of course $(P, p) \times(A, f)=\left(P \times_{B} A, p \cdot \pi_{1}\right)$. Note that, conversely, the existence of such universal arrows for all $C$ in $\mathbf{C}$ implies the existence of the right adjoint for ()$\times(A, f)$, since every object in $(\mathbf{C} \downarrow B)$ can be presented as the equalizer of two parallel morphisms of the form $\left(C \times B, \mathrm{pr}_{2}\right) \rightarrow\left(C^{\prime} \times B, \mathrm{pr}_{2}\right)$. Moreover, we have:

Theorem 3.1. $[15,22]$ For a morphism $f: A \rightarrow B$ in a finitely complete category $\mathbf{C}$, the following conditions are equivalent:

(i) $f$ is exponentiable, i.e. ( $) \times(A, f):(\mathbf{C} \downarrow B) \rightarrow(\mathbf{C} \downarrow B)$ has a right adjoint;

(ii) the 'change-of-base' functor $f^{*}:(\mathbf{C} \downarrow B) \rightarrow(\mathbf{C} \downarrow A)$ has a right adjoint;

(iii) the composite $(\mathbf{C} \downarrow B) \rightarrow \mathbf{C}$ of $f^{*}$ and the forgetful functor $(\mathbf{C} \downarrow A) \rightarrow \mathbf{C}$ has a right adjoint;

(iv) $\mathbf{C}$ has partial products of $f$ with each of its objects.

In PsRelAlg $(T)$ the pullback of $f:(X, a) \rightarrow(Y, b)$ and $g:(Z, c) \rightarrow(Y, b)$

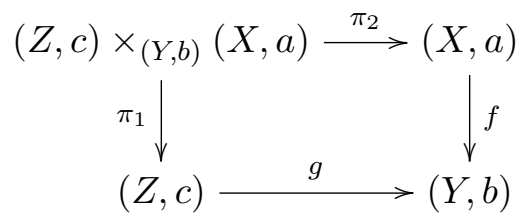

is built as in Set, that is, $Z \times_{Y} X=\{(z, x) \mid g(z)=f(x)\}$, with $\mathfrak{w} \rightarrow(z, x)$, for $\mathfrak{w} \in T\left(Z \times_{Y} X\right)$ and $(z, x) \in Z \times_{Y} X$, if $T \pi_{1}(\mathfrak{w}) \rightarrow z$ and $T \pi_{2}(\mathfrak{w}) \rightarrow x$ (see [11] for details). In [11] it was shown 
that, for $f:(X, a) \rightarrow(Y, b)$ and $(Z, c)$ in PsRelAlg $(T)$, the partial product

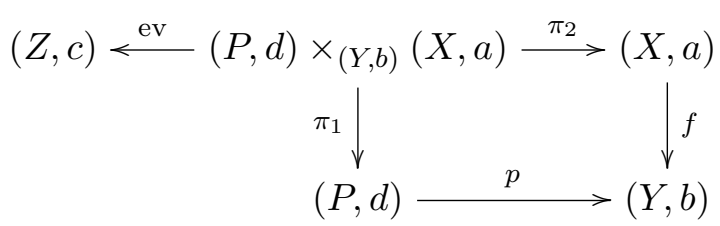

of $f$ and $Z$ can be constructed as $P=Z^{(X, f)}=\left\{(s, y) \mid y \in Y, s:\left(X_{y}, a_{y}\right) \rightarrow(Z, c)\right\}$, where $\left(X_{y}, a_{y}\right)$ is defined as the pullback

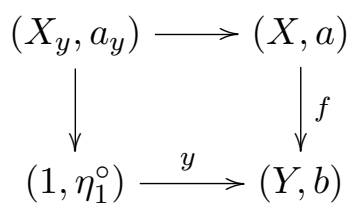

and in particular $X_{y}$ can be identified with $f^{-1}(y)$; the structure $d: T P \longrightarrow P$ on $P$ is defined, for $\mathfrak{p} \in T P$ and $(s, y) \in P$, by:

$$
\mathfrak{p} \rightarrow(s, y) \quad \text { if } \quad\left\{\begin{array}{l}
T p(\mathfrak{p}) \rightarrow y \text { and } \\
T \mathrm{ev}(\mathfrak{w}) \rightarrow \operatorname{ev}((s, y), x)=s(x), \text { whenever } \mathfrak{w} \in T\left(P \times_{Y} X\right) \text { and } x \in X \\
\text { are such that } T \pi_{1}(\mathfrak{w})=\mathfrak{p}, f(x)=y \text { and } T \pi_{2}(\mathfrak{w}) \rightarrow x
\end{array}\right.
$$

Theorem 3.2. [11] The category $\operatorname{PsRelAlg}(T)$ of pseudo-relational T-algebras is a quasitopos.

\section{Algebraic homomorphisms are exponentiable, topologically}

In this section we will prove that the partial product of an algebraic homomorphism and a relational $T$-algebra is a relational $T$-algebra, showing that algebraic homomorphisms are exponentiable in $\operatorname{RelAlg}(T)$. First we show that perfect homomorphisms, hence in particular algebraic homomorphisms, have an interpolation property which is sufficient for exponentiability (similarly to the proof for the ultrafilter monad done in [12]).

Proposition 4.1. Let $f:(X, a) \rightarrow(Y, b)$ be a perfect morphism in $\operatorname{RelA} \lg (T)$, and let the diagram

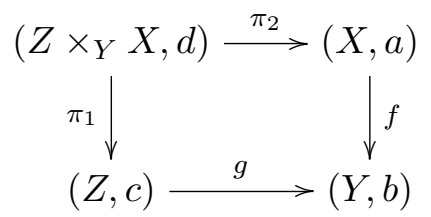

be a pullback in PsRelAlg $(T)$. Then:

(1) $\pi_{1}$ is a proper map.

(2) If $\mathfrak{W} \in T^{2}\left(Z \times_{Y} X\right)$, $(z, x) \in Z \times_{Y} X$ and $\mathfrak{Z} \in T Z$ are such that

(a) $\mu_{X}\left(T^{2} \pi_{2}(\mathfrak{W})\right) \rightarrow x$,

(b) $T^{2} \pi_{1}(\mathfrak{W}) \rightarrow \mathfrak{Z} \rightarrow z$,

then there exists $\mathfrak{w} \in T\left(Z \times_{Y} X\right)$ such that $T \pi_{1}(\mathfrak{w})=\mathfrak{Z}$ and

$$
\mathfrak{W} \rightarrow \mathfrak{w} \rightarrow(z, x) .
$$

Proof. It was proved in [11] that proper maps are pullback-stable in PsRelAlg(T), hence $\pi_{1}$ is proper, that is $\pi_{1} \cdot d=c \cdot T \pi_{1}$. Therefore, also $T \pi_{1} \cdot T d=T c \cdot T^{2} \pi_{1}$. Hence, if $T^{2} \pi_{1}(\mathfrak{W}) \rightarrow \mathfrak{Z}$, there exists $\mathfrak{w} \in T\left(Z \times_{Y} X\right)$ such that $T \pi_{1}(\mathfrak{w})=\mathfrak{Z}$ and $\mathfrak{W} \rightarrow \mathfrak{w}$. Using now the fact that $\pi_{1}$ is proper and $T \pi_{1}(\mathfrak{w})=\mathfrak{Z} \rightarrow z$, there exists $\left(z, x^{\prime}\right) \in Z \times_{Y} X$ such that $\mathfrak{w} \rightarrow\left(z, x^{\prime}\right)$. The chain 
$\mathfrak{W} \rightarrow \mathfrak{w} \rightarrow\left(z, x^{\prime}\right)$ gives rise to a chain in $X, T^{2} \pi_{2}(\mathfrak{W}) \rightarrow T \pi_{2}(\mathfrak{w}) \rightarrow x^{\prime}$, which implies that $\mu_{X}\left(T^{2} \pi_{2}(\mathfrak{W})\right) \rightarrow x^{\prime}$. Since $f(x)=f\left(x^{\prime}\right)$, perfectness of $f$ gives $x=x^{\prime}$.

Theorem 4.2. Perfect maps are exponentiable in $\operatorname{RelAlg}(T)$.

Proof. For $f:(X, a) \rightarrow(Y, b)$ perfect in $\operatorname{RelA} \lg (T)$ and $(Z, c) \in \operatorname{RelA} \lg (T)$, form the partial product $\left(p:(P, d) \rightarrow(Y, b), e:(P, d) \times_{(Y, b)}(X, a) \rightarrow(Z, c)\right)$ of $f$ and $(Z, c)$ in PsRelAlg $(T)$ as in (E). To check that $(P, d) \in \operatorname{RelAlg}(T)$, we need to check that, for

$$
\mathfrak{P} \rightarrow \mathfrak{p} \rightarrow(s, y) \text { in } P
$$

we have $\mu_{P}(\mathfrak{P}) \rightarrow(s, y)$ in $P$. The first of the two conditions defining $\mu(\mathfrak{P}) \rightarrow(s, y)$ easily follows from the fact that $(Y, b)$ belongs to $\operatorname{RelAlg}(T)$, and we will check only the second one. Suppose there exist $\tilde{\mathfrak{w}} \in T\left(P \times_{Y} X\right)$ and $x \in X$ such that $T \pi_{1}(\tilde{\mathfrak{w}})=\mu_{P}(\mathfrak{P}), f(x)=y$ and $T \pi_{2}(\tilde{\mathfrak{w}}) \rightarrow x$. Since the diagram

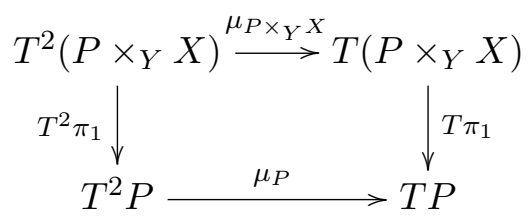

is a (BC)-square, there exists $\mathfrak{W} \in T^{2}\left(P \times_{Y} X\right)$ such that

$$
\mu_{P \times_{Y} X}(\mathfrak{W})=\tilde{\mathfrak{w}} \text { and } T^{2} \pi_{1}(\mathfrak{W})=\mathfrak{P} \text {. }
$$

The lemma above guarantees the existence of $\mathfrak{w} \in T\left(P \times_{Y} X\right)$ such that $T \pi_{1}(\mathfrak{w})=\mathfrak{p}$ and

$$
\mathfrak{W} \rightarrow \mathfrak{w} \rightarrow((s, y), x) .
$$

Therefore $T^{2} \operatorname{ev}(\mathfrak{W}) \rightarrow T$ ev $(\mathfrak{w}) \rightarrow s(x)$, hence

$$
T \operatorname{ev}(\tilde{\mathfrak{w}})=T \operatorname{ev}\left(\mu_{P \times_{Y} X}(\mathfrak{W})\right)=\mu_{Z}\left(T^{2} \operatorname{ev}(\mathfrak{W})\right) \rightarrow s(x),
$$

and so we can conclude that $\mu_{P}(\mathfrak{P}) \rightarrow(s, y)$ in $P$ by the definition of the structure on $P$.

In a different context a similar result was proved by Richter and Tholen [23].

Corollary 4.3. Algebraic homomorphisms are exponentiable in $\operatorname{RelAlg}(T)$.

\section{5. ÉtAle Algebraic homomorphisms are exponentiable, algEBraically}

In this section we will show that étale algebraic homomorphisms are exponentiable in $\mathbf{A l g}(T)$, generalizing the corresponding result for topological spaces obtained by Cagliari and Mantovani $[2]$.

Proposition 5.1. If $(X, a),(Y, b)$ and $(Z, c)$ are pseudo-relational T-algebras, $f:(X, a) \rightarrow$ $(Y, b)$ is open, and $(Y, b)$ and $(Z, c)$ are Hausdorff, then the partial product of $f$ and $(Z, c)$ in PsRelAlg $(T)$ is Hausdorff too.

Proof. Consider the partial product (E) and assume that $\mathfrak{p} \in T P$ is such that $\mathfrak{p} \rightarrow(s, y)$ and $\mathfrak{p} \rightarrow\left(s^{\prime}, y^{\prime}\right)$ in $P$. Hausdorffness of $Y$ guarantees that $y=y^{\prime}$. If $X_{y}=f^{-1}(y)$ is empty, then necessarily $s=s^{\prime}$. Otherwise, let $x \in f^{-1}(y)$. Since $T p(\mathfrak{p}) \rightarrow f(x)$, openness of $f$ gives $\mathfrak{x} \in T f^{-1}(T p(\mathfrak{p}))$ with $\mathfrak{x} \rightarrow x$. By $(\mathrm{BC})$ of $T$ there exists $\mathfrak{w} \in T\left(P \times_{Y} X\right)$ such that $T \pi_{1}(\mathfrak{w})=\mathfrak{p}$ and $T \pi_{2}(\mathfrak{w})=\mathfrak{x}$. So, in the pullback structure, $\mathfrak{w}$ is in relation with both $((s, y), x)$ and $\left(\left(s^{\prime}, y\right), x\right)$, and, consequently, $T$ ev $(\mathfrak{w})$ is related to both $s(x)$ and $s^{\prime}(x)$, which implies $s(x)=s^{\prime}(x)$ because $Z$ is Hausdorff. 
Corollary 5.2. If $f:(X, a) \rightarrow(Y, b)$ is open in $\operatorname{Haus}(T)$ and exponentiable in $\operatorname{RelAlg}(T)$, then it is exponentiable in $\operatorname{Haus}(T)$.

We remark that this result implies the corresponding result for topological spaces, due to Cagliari and Mantovani [3]. We do not know whether, as in Top, openness of $f$ is essential for its exponentiability in $\operatorname{Haus}(T)$.

Proposition 5.3. Let the diagram

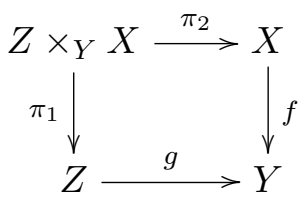

be a pullback in PsRelAlg( $(T)$, with $\pi_{1}$ a discrete fibration, $X$ and $Z$ compact, and $Y$ Hausdorff. Then the functor $T$ preserves the underlying pullback of sets.

Proof. Applying $T$ to the diagram and forming the pullback

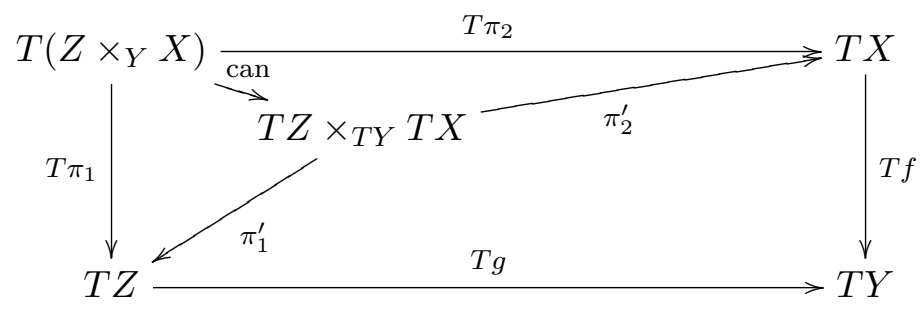

we have to show that the comparison map can : $T\left(Z \times_{Y} X\right) \rightarrow T Z \times_{T Y} T X$ is injective. Suppose $\mathfrak{w}, \mathfrak{w}^{\prime} \in T\left(Z \times_{Y} X\right)$ have $\operatorname{can}(\mathfrak{w})=\operatorname{can}\left(\mathfrak{w}^{\prime}\right)$. Denote $T \pi_{1}(\mathfrak{w})=T \pi_{1}\left(\mathfrak{w}^{\prime}\right)$ by $\mathfrak{Z}$, and $T \pi_{2}(\mathfrak{w})=T \pi_{2}\left(\mathfrak{w}^{\prime}\right)$ by $\mathfrak{x}$. By compactness of $Z$ and $X$, we can write $\mathfrak{Z} \rightarrow z$ and $\mathfrak{x} \rightarrow x$. Then Hausdorffness of $Y$ gives $g(z)=f(x)$. Hence both $\mathfrak{w}$ and $\mathfrak{w}^{\prime}$ are in relation with $(z, x)$ and are mapped, by $T \pi_{1}$, to $\mathfrak{Z}$. Since $\pi_{1}$ is a discrete fibration, $\mathfrak{w}=\mathfrak{w}^{\prime}$.

This result assures that, if $f$ is an étale algebraic homomorphism and $Z \in \mathbf{A} \lg (T)$, preservation of the pullback of the partial product (E) in PsRelAlg(T) is a necessary condition for $f$ to be exponentiable in $\operatorname{Alg}(T)$ with its exponentials built as in $\operatorname{RelAlg}(T)$. The next result shows that this pullback-preservation property is also sufficient.

Proposition 5.4. Let $f:(X, \alpha) \rightarrow(Y, \beta)$ be an étale algebraic homomorphism and $(Z, \gamma)$ a T-algebra. The domain $(P, d)$ of the partial product of $f$ and $(Z, \gamma)$ in $\operatorname{RelA} \lg (T)$ (or in PsRelAlg $(T))$ is compact if and only if $T$ preserves the pullback

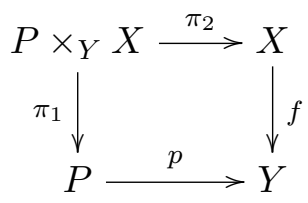

Proof. Consider again the partial product (E), assume that $T$ preserves its underlying pullback of sets, and let $\mathfrak{p} \in T P$. We need to find $(s, y) \in P$ such that $\mathfrak{p} \rightarrow(s, y)$, with $y \in Y$ and $s: X_{y} \rightarrow Z$, where $X_{y}=f^{-1}(y)$ is to be seen as

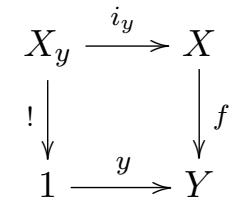


Since $Y$ is a $T$-algebra, there is a unique $y \in Y$ such that $T p(\mathfrak{p}) \rightarrow y$. Hence, we are left with the construction of $s: X_{y} \rightarrow Z$.

Since $f$ is étale, the diagram

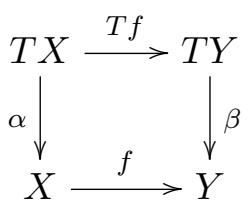

is a pullback in Set, and so it determines a pullback in $\operatorname{Alg}(T)$ (where $T X$ and $T Y$ have the free algebra structures) and therefore in $\operatorname{RelA} \lg (T)$. Hence we can consider the following commutative diagram, where both squares 1 and 2 are pullbacks.

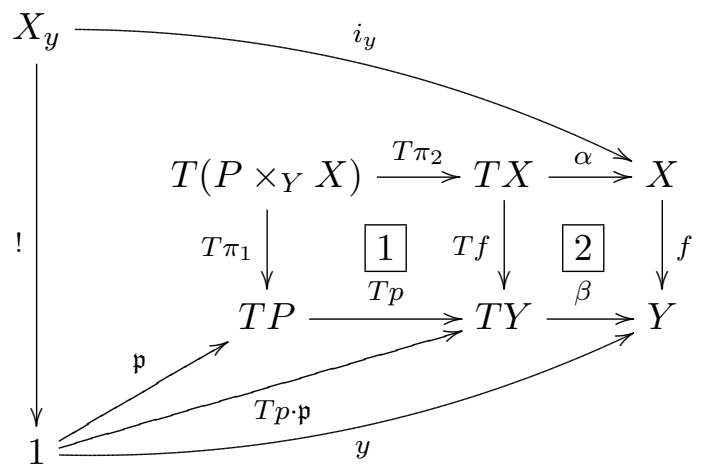

Since the diagram 1122 is a pullback, there exists a (unique) map $\kappa: X_{y} \rightarrow T\left(P \times_{Y} X\right)$ with $T \pi_{1}(\kappa(x))=\mathfrak{p}$ and $\alpha\left(T \pi_{2}(\kappa(x))\right)=x$, for each $x \in X_{y}$. Using this map, we define $s$ as the composite

$$
X_{y} \stackrel{\kappa}{\longrightarrow} T\left(P \times_{Y} X\right) \stackrel{T \mathrm{ev}}{\longrightarrow} T Z \stackrel{\gamma}{\longrightarrow} Z .
$$

To prove that $\mathfrak{p} \rightarrow(s, y)$, we need to show that $T \operatorname{ev}(\mathfrak{w}) \rightarrow s(x)$ whenever $\mathfrak{w} \in T\left(P \times_{Y} X\right)$ and $x \in X$ have $T \pi_{1}(\mathfrak{w})=\mathfrak{p}, f(x)=y$, and $T \pi_{2}(\mathfrak{w}) \rightarrow x$. Since $(X, \alpha)$ is a $T$-algebra, $T \pi_{2}(\mathfrak{w}) \rightarrow x$ means $\alpha\left(T \pi_{2}(\mathfrak{w})\right)=x$, and since $1 / 2$ is a pullback, this equality, together with $T \pi_{1}(\mathfrak{w})=\mathfrak{p}$, imply $\mathfrak{w}=\kappa(x)$. This gives $s(x)=\gamma(T \operatorname{ev}(\kappa(x)))=\gamma(T \operatorname{ev}(\mathfrak{w}))$, and so $T$ ev $(\mathfrak{w}) \rightarrow s(x)$ in the $T$-algebra $(Z, \gamma)$, as desired.

Theorem 5.5. Every étale algebraic homomorphism is exponentiable in $\operatorname{Alg}(T)$, with the exponentials built as in $\operatorname{RelAlg}(T)$.

Proof. It follows directly from Theorem 2.1, Corollary 4.3 and Propositions 5.1 and 5.4.

\section{EXAmples}

6.1 Compact Hausdorff spaces. In case $T=(T, \eta, \mu)$ is the ultrafilter monad, both $T$ and $\mu$ satisfy (BC). Hence Theorem 5.5 says that an étale continuous map between compact Hausdorff spaces is exponentiable. Cagliari and Mantovani [2] showed that also the reverse implication holds, that is, an exponentiable continuous map in CompHaus is étale. Moreover, the exponentials coincide with the exponentials built in Top.

6.2 Sup-lattices. When $T=(P, \eta, \mu)$ is the powerset monad, again both $P$ and $\mu$ satisfy (BC). $\mathbf{A} \lg (P)$ is the category Sup of complete lattices and sup-preserving maps. Theorem 5.5 says that étale homomorphisms are exponentiable in Sup. In fact étale and exponentiable homomorphisms coincide, as shown next. 
Proposition. For a morphism $f: X \rightarrow Y$ in Sup, the following conditions are equivalent:

(i) $f$ is exponentiable;

(ii) the diagram

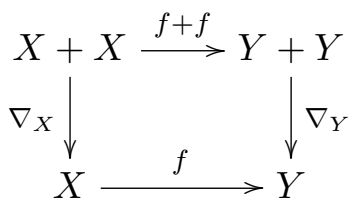

is a pullback;

(iii) for all $x \in X$ and $y^{\prime} \in Y$ with $y^{\prime} \leq y=f(x)$, there is a unique $x^{\prime} \in X$ with $x^{\prime} \leq x$ and $f\left(x^{\prime}\right)=y^{\prime}$

(iv) $f$ is a down-closed embedding;

(v) $f$ is étale.

Proof. We know that (v) $\Rightarrow$ (i), by Theorem 5.5, and that (i) $\Rightarrow$ (ii) since pulling back along an exponentiable morphism preserves colimits.

(ii) $\Rightarrow$ (iii): Since Sup admits an enrichment in the category of commutative monoids via the $\checkmark$ operation, its finite coproducts are canonically isomorphic to products, and the codiagonal $\nabla_{X}: X+X \rightarrow X$ is given by $\nabla_{X}\left(x, x^{\prime}\right)=x \vee x^{\prime}$. Let $x \in X$ and $y^{\prime} \in Y$ be such that $y^{\prime} \leq y=f(x)$. Then $y=y^{\prime} \vee y$, hence there is a (unique) pair $\left(x^{\prime}, x^{\prime \prime}\right)$ with $f\left(x^{\prime}\right)=y^{\prime}, f\left(x^{\prime \prime}\right)=y$ and $x^{\prime} \vee x^{\prime \prime}=x$, hence $x^{\prime} \leq x$. By uniqueness, $x^{\prime \prime}=x$ and $x^{\prime}$ is indeed unique.

To conclude that (iii) $\Rightarrow$ (iv) we only have to check that $f$ is injective, which follows easily from the uniqueness of $x^{\prime}$ above.

(iv) $\Rightarrow(v)$ : Since down-closed embeddings are pullback-stable, we only have to show that every down-closed embedding is a discrete fibration. A morphism $f: X \rightarrow Y$ in Sup is a discrete fibration if and only if

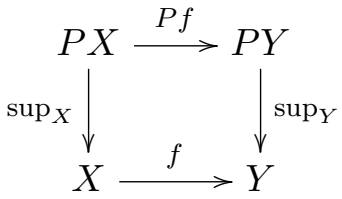

is a pullback. If $f$ is a down-closed embedding, and $x=\sup S$, for $x \in X$ and $S \subseteq Y$, then $S \subseteq X$ and the diagram is a pullback.

The category Inf of complete lattices and inf-preserving maps is isomorphic to Sup, via

$$
f: X \rightarrow Y \mapsto f^{\mathrm{op}}: X^{\mathrm{op}} \rightarrow Y^{\mathrm{op}} .
$$

Hence we can conclude that, in Inf, a morphism $f: X \rightarrow Y$ is exponentiable if and only if it is an up-closed embedding.

6.3 Continuous lattices. For the filter monad $F=(F, \eta, \mu), \mathbf{A} \lg (F)$ is the category ContLat of continuous lattices and monotone maps preserving infima and directed suprema (see [14]). Since $F$ and $\mu$ satisfy (BC), Theorem 5.5 applies, that is every étale homomorphism is exponentiable in ContLat.

Lemma. For a continuous lattice $Y$ and $y \in Y$, the following conditions are equivalent:

(i) the embedding $\uparrow y \rightarrow Y$ is étale;

(ii) $y$ is compact. 
Proof. (i) $\Rightarrow$ (ii): Let $\mathfrak{f}=\langle\{\uparrow x \mid x \ll y\}\rangle$, where $\ll$ is the way below relation. Then $y=$ $\sup _{B \in \mathfrak{f}} \inf B$. Hence, $\uparrow y \in \mathfrak{f}$, that is, $\uparrow y \supseteq \uparrow x$ for some $x \ll x$. But this implies $x \geq y$, hence $x=y$ and $y \ll y$.

(ii) $\Rightarrow$ (i): Let $\mathfrak{g}$ be a filter on $Y$ with $y=\sup _{B \in \mathfrak{g}} \inf B$. Since $y \ll y, y \leq \inf B$ for some $B \in \mathfrak{g}$, hence $B \subseteq \uparrow y$ and therefore $\uparrow y \in \mathfrak{g}$, which, together with the preservation of infima and directed suprema by the inclusion $\uparrow y \rightarrow Y$, gives that this morphism is étale.

The arguments used in the proof of Proposition 6 can be used here, since ContLat is also enriched in the category of commutative monoids via the $\wedge$ operation. Hence:

(1) every exponentiable morphism in ContLat is an up-closed embedding, that is, it is (up to isomorphism) an inclusion $\uparrow y \rightarrow Y$, for some $y \in Y$;

(2) a morphism is étale if and only if it is, up to isomorphism, an inclusion $\uparrow y \rightarrow Y$ with $y$ compact.

Therefore one has:

Proposition. For a morphism $f: X \rightarrow Y$ in ContLat, each of the conditions below implies the following one:

(i) there is a compact element $y$ of $Y$ such that $f$ is, up to isomorphism, the inclusion $\uparrow y \rightarrow Y$;

(ii) $f$ is exponentiable;

(iii) there is an element $y$ of $Y$ such that $f$ is, up to isomorphism, the inclusion $\uparrow y \rightarrow Y$.

We do not know whether exponentiability of $\uparrow y \rightarrow Y$ implies compactness of $y$.

6.4 Monoids. Let $M=(M, \eta, \mu)$ be the free-monoid monad on Set, with $M X$ the set of words in the alphabet $X$ (of length $\geq 0$ ), $\eta_{X}$ the insertion of $X$ into $M X$ as one-letter words, and concatenation $\mu_{X}$. It is well known that $\operatorname{Alg}(M)$ is the category Mon of monoids and monoid homomorphisms. This monad is cartesian, hence in particular both $M$ and $\mu$ satisfy (BC).

We will show next that in Mon étale homomorphisms are exactly the exponentiable ones.

It follows from Theorem 5.5 that étale homomorphisms are exponentiable since the free-monoid monad satisfies (BC).

To prove the converse, let $f:(X, \alpha) \rightarrow(Y, \beta)$ be an exponentiable homomorphism in Mon, and consider the commutative diagram

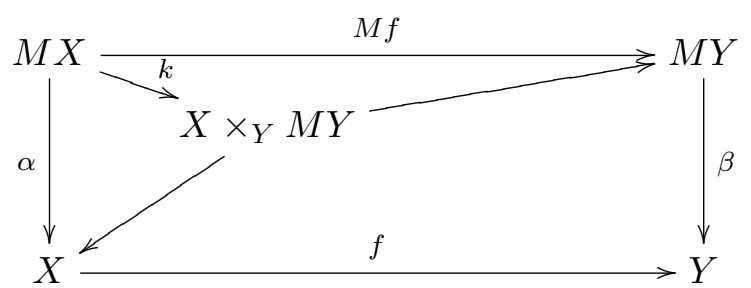

We will show that the canonical map $k$ is injective and surjective, that is $f$ is a discrete fibration, which, by Corollary 2.2, gives that $f$ is étale.

Injectivity. First we observe that exponentiability of $f$ implies preservation of the initial object by the functor $f^{*}$, which means that the kernel $\operatorname{Ker}(f)$ of $f$ is a trivial monoid. Now, suppose $k\left(x_{1}, \cdots, x_{n}\right)=k\left(x_{1}^{\prime}, \cdots, x_{n^{\prime}}^{\prime}\right)$. This means

$$
x_{1} \cdots x_{n}=x_{1}^{\prime} \cdots x_{n^{\prime}}^{\prime} \text { in } X, \text { and }\left(f\left(x_{1}\right), \cdots, f\left(x_{n}\right)\right)=\left(f\left(x_{1}^{\prime}\right), \cdots, f\left(x_{n^{\prime}}^{\prime}\right)\right) \text { in } M Y,
$$


which implies $n=n^{\prime}$, and is equivalent to

$$
x_{1} \cdots x_{n}=x_{1}^{\prime} \cdots x_{n}^{\prime} \text { in } X \text {, and } f\left(x_{i}\right)=f\left(x_{i}^{\prime}\right) \text { in } Y \text { for each } i=1, \cdots, n .
$$

If $f\left(x_{i}\right)=f\left(x_{i}^{\prime}\right)=1$ for some $i$, then $x_{i}=x_{i}^{\prime}=1$ since $\operatorname{Ker}(f)=\{1\}$, and simultaneously removing $x_{i}$ from the sequence $\left(x_{1}, \cdots, x_{n}\right)$ and $x_{i}^{\prime}$ from the sequence $\left(x_{1}^{\prime}, \cdots, x_{n}^{\prime}\right)$ will not change anything in $(\mathrm{G})$ except decreasing $n$. Therefore, without loss of generality, we can assume that $f\left(x_{i}\right) \neq 1 \neq f\left(x_{i}^{\prime}\right)$, and so $x_{i} \neq 1 \neq x_{i}^{\prime}$ for each $i$. Let $\iota_{1}, \iota_{2}: X \rightarrow X+X$ and $\kappa_{1}, \kappa_{2}: Y \rightarrow Y+Y$ be the coproduct injections. Consider the elements

$$
t=\iota_{\varepsilon_{1}}\left(x_{1}\right) \cdots \iota_{\varepsilon_{n}}\left(x_{n}\right) \text { and } t^{\prime}=\iota_{\varepsilon_{1}}\left(x_{1}^{\prime}\right) \cdots \iota_{\varepsilon_{n}}\left(x_{n}^{\prime}\right) \in X+X
$$

where $\varepsilon_{i}=1$ for even $i$ and $\varepsilon_{i}=2$ for odd $i$; their images in $Y+Y$ under $f+f$ are

$$
(f+f)(t)=\kappa_{\varepsilon_{1}}\left(f\left(x_{1}\right)\right) \cdots \kappa_{\varepsilon_{n}}\left(f\left(x_{n}\right)\right) \text { and }(f+f)\left(t^{\prime}\right)=\kappa_{\varepsilon_{1}}\left(f\left(x_{1}^{\prime}\right)\right) \cdots \kappa_{\varepsilon_{n}}\left(f\left(x_{n}^{\prime}\right)\right),
$$

respectively. Next, consider diagram (F) as in Proposition 6.2, which is a pullback again since $f$ is exponentiable. In this diagram, as follows from $(\mathrm{G})$ and $(\mathrm{H})$, the elements $t$ and $t^{\prime}$ have the same images in $X$ and in $Y+Y$. Therefore $t=t^{\prime}$, and since $x_{i} \neq 1 \neq x_{i}^{\prime}$ for each $i$, this implies $\left(x_{1}, \cdots, x_{n}\right)=\left(x_{1}^{\prime}, \cdots, x_{n^{\prime}}^{\prime}\right)$. That is, $k$ is injective.

Surjectivity. We have to prove that, for every $x \in X$ and every $\left(y_{1}, \cdots, y_{n}\right) \in M Y$ with $f(x)=y_{1} \cdots y_{n}$, there exist $\left(x_{1}, \cdots, x_{n}\right) \in M X$ with $x_{1} \cdots x_{n}=x$ in $X$ and $f\left(x_{i}\right)=y_{i}$ for each $i$. Again, if $y_{i}=1$ for some $i$, removing it will not change the equality $f(x)=y_{1} \cdots y_{n}$, and so we can assume that $y_{i} \neq 1$ for each $i$. Next, in the diagram (F) we have $f(x)=\nabla_{Y}\left(\kappa_{\varepsilon_{1}}\left(y_{1}\right) \cdots \kappa_{\varepsilon_{n}}\left(y_{n}\right)\right)$ in the notation above. Since (F) is a pullback diagram, and having in mind the construction of coproducts of monoids and the fact that $y_{i} \neq 1$ for each $i$, we conclude that there a sequence $\left(x_{1}, \cdots, x_{n}\right)$ of elements in $X$ with the desired properties.

That is, $f$ is exponentiable if and only if it is étale.

Let us make some further remarks about monoids. As mentioned above, whenever $f$ is exponentiable, we have $\operatorname{Ker}(f)=\{1\}$. More generally, the same is true whenever $f$ is open. This immediately follows from the fact that $\operatorname{Ker}(M f)=\{1\}$ for any $f$. However, even if $f$ étale, it does not have to be injective. Note that, for every map $f$ from a set $X$ to a set $Y$, since $M$ is a cartesian monad, the diagram

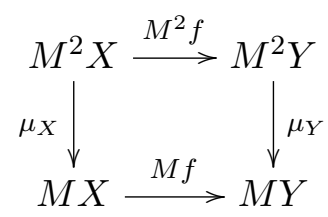

is a pullback, and so $M f:\left(M X, \mu_{X}\right) \rightarrow\left(M Y, \mu_{Y}\right)$ is étale. On the other hand, injectivity of an algebraic homomorphism is not sufficient for being étale, and not even for being open. For example, for an additive monoid $\mathbb{N}$ of natural numbers, the inclusion homomorphism $\mathbb{N} \backslash\{1\} \rightarrow \mathbb{N}$ is not open simply because $1+1=2 \in \mathbb{N} \backslash\{1\}$, while $1 \notin \mathbb{N} \backslash\{1\}$.

6.5 Semigroups. If we take instead the monad $M^{\prime}$ of non-empty words, $\operatorname{Alg}\left(M^{\prime}\right)$ is the category SGrp of semigroups and semigroup homomorphisms. An argument analogous to the previous one, used for monoids, shows that exponentiable homomorphisms in SGrp are exactly the étale homomorphisms.

6.6 $M$-Sets. For a monoid $M=(M, \cdot, e)$, consider the free $M$-set monad $T=(M \times-, \eta, \mu)$, where $\eta_{X}=\left\langle e, 1_{X}\right\rangle: X \rightarrow M \times X$, and $\mu_{X}: M \times M \times X \rightarrow M \times X$ is given by $\mu_{X}(m, n, x)=$ $(m \cdot n, x)$. Then $\operatorname{Alg}(T)$ is the category $M$-Set of $M$-sets, that is, sets $X$ equipped with an 
$M$-action, and equivariant maps. Again, since the monad is cartesian, $M \times-$ and $\mu$ satisfy (BC). The extension $\bar{T}: \mathbf{R e l} \rightarrow \mathbf{R e l}$ is defined, for a relation $r: X \rightarrow Y$, by

$$
(m, x) \bar{T} r(n, y) \Leftrightarrow m=n \text { and } x r y .
$$

To describe a relational $T$-algebra $a: M \times X \mapsto X$, we write $x \stackrel{n}{\rightarrow} y$ if $(n, x) a y$. Then $a$ : $M \times X \mapsto X$ is a relational algebra if and only if, for all $x, y, z \in X$ :

(a) $x \stackrel{e}{\rightarrow} x$, and

(b) $x \stackrel{n}{\rightarrow} y$ and $y \stackrel{m}{\rightarrow} z \Rightarrow x \stackrel{m \cdot n}{\rightarrow} z$.

A homomorphism $f:(X, a) \rightarrow(Y, b)$ is a map $f: X \rightarrow Y$ such that, for all $x, x^{\prime} \in X$,

(c) $x \stackrel{n}{\rightarrow} x^{\prime} \Rightarrow f(x) \stackrel{n}{\rightarrow} f\left(x^{\prime}\right)$.

Therefore relational $T$-algebras can be seen as $M$-labeled ordered sets and homomorphisms as monotone maps.

The category $M$-Set is a topos, hence it is locally cartesian closed, so that every homomorphism is exponentiable in $M$-Set. However, in general there are homomorphisms in $M$-Set which are not étale. Consider for instance $M=(\mathbb{N}, \times, 1)$ and the action of $\mathbb{N}$ on $\mathbb{Z}$ and $\mathbb{Q}$ via multiplication. The inclusion

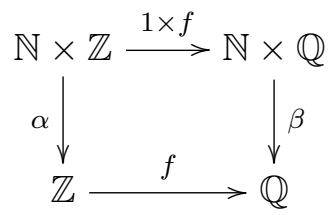

is not open, since $f(1)=1=\beta\left(2, \frac{1}{2}\right)$ and there is no element of $\mathbb{N} \times \mathbb{Z}$ mapped by $1 \times f$ into $\left(2, \frac{1}{2}\right)$.

The case of $M$-sets is worth to study in detail. Theorem 5.5 states that étale maps are exponentiable in $M$-Set, with exponentials built as in the category of $M$-labeled sets and monotone maps. In fact, although any map is exponentiable in $M$-Set, the exponentials are built as in $M$ labeled ordered sets if and only if the map is étale, as we show next. Note that $f:(X, \alpha) \rightarrow(Y, \beta)$ in $M$-Set is étale if and only if

$$
(\forall x \in X)(\forall m \in M)(\forall y \in Y) f(x)=m y \Rightarrow\left(\exists ! x^{\prime} \in X\right) m x^{\prime}=x \text { and } f\left(x^{\prime}\right)=y \text {. }
$$

Now, we are going to check that the partial product of $f:(X, \alpha) \rightarrow(Y, \beta) \in M$-Set and $(Z, \gamma) \in M$-Set calculated in $\operatorname{RelAlg}(T)$ (as described in Section 3) is an $M$-set only if $f$ is étale. First we remark that, for each $y \in Y$, the relational structure $\alpha_{y}$ on $X_{y}$, obtained by pulling back $y:\left(1, \eta_{1}^{\circ}\right) \rightarrow(Y, \beta)$ along $f$, is discrete, that is $x \stackrel{m}{\rightarrow} x^{\prime}$, if and only if $m=e$ and $x=x^{\prime}$. Hence any map $s: X_{y} \rightarrow Z$ becomes a homomorphism $s:\left(X_{y}, \alpha_{y}\right) \rightarrow(Z, \gamma)$; that is,

$$
P=Z^{(X, f)}=\left\{(s, y) \mid y \in Y, s: X_{y} \rightarrow Z \text { a map }\right\} .
$$

The relational $T$-algebra structure on $P$ has

$$
(s, y) \stackrel{m}{\rightarrow}\left(s^{\prime}, y^{\prime}\right) \Leftrightarrow\left\{\begin{array}{l}
m y=y^{\prime}, \text { and } \\
m(s(x))=s^{\prime}\left(x^{\prime}\right), \text { whenever } f\left(x^{\prime}\right)=y^{\prime} \text { and } m x=x^{\prime},
\end{array}\right.
$$

which is the direct translation of its general description in Section 3 to the present case. It shows that, whenever $Z$ has more than one element, the uniqueness of $\left(s^{\prime}, y^{\prime}\right)$ satisfying $(s, y) \stackrel{m}{\rightarrow}\left(s^{\prime}, y^{\prime}\right)$ is equivalent to the existence and uniqueness of $x \in X$ satisfying $f(x)=y$ and $m x=x^{\prime}$ for each 
$y \in Y$ and $x^{\prime} \in X$ with $f\left(x^{\prime}\right)=m y$. That is, the partial product of $f$ with every $T$-algebra is a $T$-algebra if and only if $f$ is étale.

6.7 $G$-Sets. In the previous example, if $M$ is a group $G$, then every morphism in $G$-Set is étale. Indeed, for every homomorphism $f:(X, \alpha) \rightarrow(Y, \beta)$, given $x \in X$ and $(g, y) \in G \times Y$ such that $f(x)=g y$, put $x^{\prime}=g^{-1} x$. Then $\left(g, x^{\prime}\right)$ is the unique element of $G \times X$ such that $g x^{\prime}=x$ and $\left(1_{G} \times f\right)\left(g, x^{\prime}\right)=\left(g, g^{-1} f(x)\right)=(g, y)$. Hence, exponentiable and étale homomorphisms coincide.

6.8 Remark. In this paper we only considered monads on Set. However, the results of $[4,5]$ on exponentiable morphisms in categories of domains suggest that our results, suitably adapted, hold for monads on Ord and other categories. For instance, from [4, Theorem 1.9 and Lemma 1.5] it follows that a monomorphism $f: X \rightarrow B$ in the category DCPO of directed-complete ordered sets and continuous maps is exponentiable in DCPO if and only if $f$ is exponentiable in Ord and, moreover, for every $x \in X$ and every directed down-set $D \subseteq B$ with $f(x)=\bigvee D$, there is a directed subset $C \subseteq X$ with $x=\bigvee C$ and $f(C) \subseteq D$ is cofinal.

\section{REFERENCES}

[1] M. Barr, Relational algebras, in: Reports of the Midwest Category Seminar, IV, Lecture Notes in Mathematics, Vol. 137. Springer, Berlin, 1970, pp. 39-55.

[2] F. Cagliari and S. Mantovani, Local homeomorphisms as the exponentiable morphisms in compact Hausdorff spaces, Topology Appl. 41 (1991), 263-272.

[3] F. Cagliari and S. Mantovani, Preservation of topological properties under exponentiation, Topology Appl. 47 (1992), 149-159.

[4] F. Cagliari and S. Mantovani, Exponentiable monomorphisms in categories of domains, J. Pure Appl. Algebra 211 (2007), 404-413.

[5] F. Cagliari and S. Mantovani, Exponentiable morphisms of domains, Math. Structures Comput. Sci. 18 (2008), 1005-1016.

[6] M. M. Clementino and D. Hofmann, Triquotient maps via ultrafilter convergence, Proc. Amer. Math. Soc. 130 (2002), 3423-3431.

[7] M. M. Clementino and D. Hofmann, Topological features of lax algebras, Appl. Categ. Structures 11 (2003), 267-286.

[8] M. M. Clementino and D. Hofmann, On extensions of lax monads, Theory Appl. Categories 13 (2004), 41-60.

[9] M. M. Clementino and D. Hofmann, Effective descent morphisms in categories of lax algebras, Appl. Categ. Structures 12 (2004), 413-425.

[10] M. M. Clementino, D. Hofmann and G. Janelidze, Local homeomorphisms via ultrafilter convergence, Proc. Amer. Math. Soc. 133 (2005), 917-922.

[11] M. M. Clementino, D. Hofmann and W. Tholen, Exponentiability in categories of lax algebras, Theory Appl. Categories 11 (2003), 337-352.

[12] M. M. Clementino, D. Hofmann and W. Tholen, The convergence approach to exponentiable maps, Port. Math. 60 (2003), 139-160.

[13] M. M. Clementino and W. Tholen, Metric, topology and multicategory - a common approach, J. Pure Appl. Algebra 179 (2003), 13-47.

[14] A. Day, Filter monads, continuous lattices and closure systems, Canad. J. Math. XXVII (1975), 50-59.

[15] R. Dyckhoff and W. Tholen, Exponentiable morphisms, partial products and pullback complements, J. Pure Appl. Algebra 49 (1987), 103-116.

[16] P. T. Johnstone, Collapsed toposes and cartesian closed varieties, J. Algebra 129 (1990), 446-480.

[17] F. W. Lawvere, Functorial semantics of algebraic theories, Dissertation, Columbia University 1963; Reprints in Theory and Applications of Categories 5 (2004), pp. 1-121.

[18] M. Mahmoudi, C. Schubert and W. Tholen, Universality of coproducts in categories of lax algebras, Appl. Categ. Structures 14 (2006), 243-249.

[19] E.G. Manes, A triple theoretic construction of compact algebras, in: Lecture Notes in Math. 80 (Springer, Berlin 1969) pp. 91-118. 
[20] E.G. Manes, Compact Hausdorff objects, Topology Appl. 4 (1974), 341-360.

[21] E. Manes, Taut monads and T0-spaces, Theoret. Comput. Sci. 275 (2002), 79-109

[22] S. Niefield, Cartesianness: topological spaces, uniform spaces, and affine schemes, J. Pure Appl. Algebra 23 (1982) 147-167.

[23] G. Richter, W. Tholen, Perfect maps are exponentiable - categorically, Theory Appl. Categ. 8 (2001), 457-464.

CMuC, Department of Mathematics, University of Coimbra, 3001-454 Coimbra, Portugal

E-mail address: mmc@mat.uc.pt

Departamento de Matemática, Universidade de Aveiro, 3810-193 Aveiro, Portugal

E-mail address: dirk@ua.pt

Department of Mathematics and Applied Mathematics, University of Cape Town, Rondebosch 7701, Cape Town, South Africa

E-mail address: George.Janelidze@uct.ac.za 